\title{
ARTICLE \\ Optogenetic reactivation of prefrontal social neural ensembles mimics social buffering of fear
}

\author{
Vanessa A. Gutzeit ${ }^{1}$, Kylia Ahuna ${ }^{2}$, Tabia L. Santos ${ }^{3}$, Ashley M. Cunningham ${ }^{4}$, Meghin Sadsad Rooney ${ }^{5}$, Andrea Muñoz Zamora ${ }^{6,7}$, \\ Christine A. Denny ${ }^{6,7}$ and Zoe R. Donaldson iD $^{2,8}$
}

\begin{abstract}
Social buffering occurs when the presence of a companion attenuates the physiological and/or behavioral effects of a stressful or fear-provoking event. It represents a way in which social interactions can immediately and potently modulate behavior. As such, social buffering is one mechanism by which strong social support increases resilience to mental illness. Although the behavioral and neuroendocrine impacts of social buffering are well studied in multiple species, including humans, the neuronal underpinnings of this behavioral phenomenon remain largely unexplored. Previous work has shown that the infralimbic prefrontal cortex (IL-PFC) is important for processing social information and, in separate studies, for modulating fear and anxiety. Thus, we hypothesized that socially active cells within the IL-PFC may integrate social information to modulate fear responsivity. To test this hypothesis, we employed social buffering paradigms in male and female mice. Similar to prior studies in rats, we found that the presence of a cagemate reduced freezing in fear- and anxiety-provoking contexts. In accordance with previous work, we demonstrated that interaction with a novel or familiar conspecific induces activity in the IL-PFC as evidenced by increased immediate early gene (IEG) expression. We then utilized an activity-dependent tagging murine line, the ArcCreER ${ }^{\mathrm{T} 2}$ mice, to express channelrhodopsin (ChR2) in neurons active during the social encoding of a new cagemate. We found that optogenetic reactivation of these socially active neuronal ensembles phenocopied the effects of cagemate presence in male and female mice in learned and innate fear contexts without being inherently rewarding or altering locomotion. These data suggest that a social neural ensemble within the IL-PFC may contribute to social buffering of fear. These neurons may represent a novel therapeutic target for fear and anxiety disorders.
\end{abstract}

Neuropsychopharmacology (2020) 45:1068-1077; https://doi.org/10.1038/s41386-020-0631-1

\section{INTRODUCTION}

Strong social support in humans is intricately intertwined with our health and well-being [1-3]. The presence of strong social relationships decreases one's likelihood to develop mental illness and enhances our ability to recover from heart attacks, cancer, and other illnesses [4-7]. In contrast, social isolation has been extensively linked with deleterious health outcomes, ranging from increased rates of coronary heart disease and stroke to neurological and mental health disorders [8]. Adults who are socially isolated or unhappy about their social relationships are at an increased risk of premature mortality, comparable to the risk conferred by obesity or lack of physical activity [2], a finding that has also been observed among other non-human primates that form complex social relationships [9]. Together, these epidemiological findings suggest that healthy social relationships have multiple beneficial functions, whereas isolation or abusive relationships are deleterious.

One proposed mechanism by which positive social interactions modulate health is by reducing the behavioral, physiological, and neural responses to stress or threat, a phenomenon referred to as social buffering $[10,11]$. Social buffering is highly conserved across taxa $[12,13]$ and has been documented in rats [14, 15], zebrafish [16], goats [17], pigs [18], non-human primates [19-23], and humans [24]. In humans, social buffering consistently attenuates behavioral fear responses and anxiety levels in both experimental and self-reported contexts [25]. Squirrel monkeys exhibited ameliorated stress responses after exposure to a fearful stimulus (a snake), if social companions were present during exposure [26]. In rats, stress responses to fearful stimuli were reduced in the presence of a same-sex partner $[27,28]$ and in mice the presence of cagemates reduced anxiolytic responses to novel and aversive stimuli.

Given the highly conserved nature of social buffering, it is hypothesized that the underlying neural mechanisms are also shared across taxa $[12,29]$. In particular, the prefrontal cortex (PFC) modulates social behavior [30-32], fear responses $[33,34]$, and resilience to stressors [35-37] in many different species. Critically, inhibition of the PFC with the GABA agonist, muscimol, blocks the effects of social familiarity in reducing anxiety-like behaviors [38], suggesting a potentially direct role for the PFC in social buffering of fear and/or anxiety. However, the PFC is a heterogeneous brain structure and the

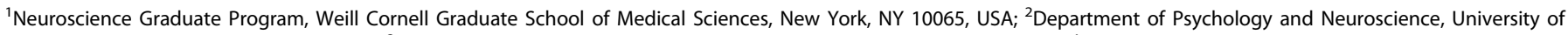

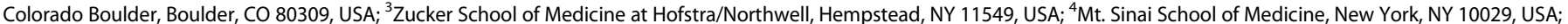

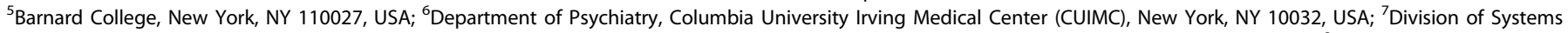

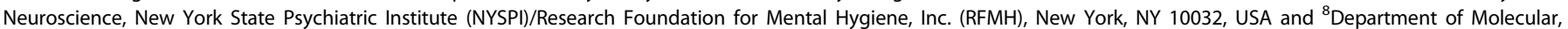
Cellular and Developmental Biology, University of Colorado Boulder, Boulder, CO 80309, USA
}

Correspondence: Zoe R. Donaldson (zoe.donaldson@colorado.edu)

Received: 4 September 2019 Revised: 23 January 2020 Accepted: 28 January 2020

Published online: 8 February 2020 
specific neuronal populations that underlie social buffering remain largely unknown.

Previous work suggests that neuronal ensembles consisting of sparse, interspersed cells mediate distinct behaviors [39]. These neuronal ensembles are often referred to as memory traces or engrams [40, 41]. Social engrams in the hippocampus (HPC) modulate social recognition memory [42], and memory traces in the ventromedial hypothalamus encode social fear [43], suggesting that socially active neurons in different brain regions encode different aspects of social behavior and social experience. In addition to its role in social behavior, the PFC is also critical for fear learning and extinction [34, 44-46], both of which are sensitive to conspecific presence in mice [47]. Further evidence suggests that extinction learning (albeit for drug self-administration) is encoded in discrete neuronal ensembles in the PFC [48]. Thus, given the role of the PFC in social buffering and fear learning/extinction, we asked whether socially active (social) neuronal ensembles within this brain region integrate social experience and were sufficient to modulate fear and anxiety.

Here we first validated behavioral metrics of social buffering of fear in mice using a paradigm similar to that previously employed in rats [15]. We subsequently showed that social interaction with a novel or familiar cagemate results in increased protein expression of the immediate early gene (IEG), c-fos, in the infralimbic PFC (IL-PFC). Then, to gain genetic access to socially active neurons in the IL-PFC, we used the ArcCreER ${ }^{\mathrm{T} 2}$ mice. This mouse line takes advantage of the IEG promoter Arc to indelibly label active neuronal populations in a temporally specific manner [49]. Using the ArcCreER $^{\mathrm{T} 2}$ mice, we tagged neurons that were active during the introduction of a new cagemate with ChR2-enhanced yellow fluorescent protein (eYFP). Subsequent optogenetic activation of the labeled social neural ensemble reduced freezing in innate and learned fear tasks, mimicking the behavioral effects of social buffering without altering locomotion or reward/aversion. These data suggest that social neural ensembles in the IL-PFC may mediate the effects of social buffering. Given that social buffering remains one of the most potent natural regulators of fear and anxiety, targeting this cell population represents a novel therapeutic opportunity.

\section{MATERIALS AND METHODS}

\section{Animals}

All mice were bred in-house using a mixed background consisting mostly of $129 \mathrm{~S} 6 / \mathrm{SvEv}$ with a small amount of C57BI/6J. For all experiments, mice were generated by crossing $\operatorname{ArcCreER}^{T 2}(+)$ mice [49] with Ai32 heterozygous or homozygous mice (Rosa ${ }^{\text {ChR2-eYFP }}$ or Rosa ChR2-eYFP/ChR2-eYFP) obtained from Jackson Lab [50]. These mice contain a STOP-floxed ChR2-eYFP integrated at the ROSA locus, resulting in ArcCreER ${ }^{\text {T2 }}$ ::Rosa ${ }^{\text {ChR2-eYFP }}$, ArcCreER ${ }^{\text {T2 }}$, Rosa ${ }^{\text {ChR2-eYFP }}$, and wild-type pups. These two mouse lines have been extensively validated [49-51]. This ensured that all mice had the same genetic background across experiments. Genotyping was performed as previously described [49, 50, 52]). Male and female mice began experimental testing between post-natal day (PND) 55 and PND 95 (ferrule implantation in optogenetic experiments occurred up to 14 days prior to this).

For social buffering experiments, female companion mice consisted of $\mathrm{C} 57 \mathrm{BI} / 6 \mathrm{~J}$ mice from Jackson Laboratories, which were ovariectomized in-house upon arrival, allowed to recover for at least 2 weeks, and used until they reached 1 year of age. The same mice were used as companions across multiple experiments.

Mice were housed four to five per cage until experiments began. All cages containing mice in active experiments were changed by experimenters and all cage changes occurred at least $48 \mathrm{~h}$ prior to behavioral testing. Mice were maintained on a 12:12 h light:dark cycle $\left(06: 00-18: 00 \mathrm{~h}\right.$ lights on) at $21-25^{\circ} \mathrm{C}$ with ad libitum food and water. All behavioral experiments took place during the light cycle. All animal procedures were approved by the New York State Psychiatric Institute's and University of Colorado's Institutional Animal Care and Use Committees.

Social buffering of fear responses

To assess the effects of conspecific presence on freezing in fearprovoking environments, we conducted a series of behavioral tests (detailed experimental procedures available in Supplementary Methods). Experimental mice were singly housed in fresh cages for $48 \mathrm{~h}$ and then provided with an ovariectomized female cagemate for the remainder of the study. In the first series of behavioral tests, mice were fear conditioned (FC) 5 days after cagemate introduction. Freezing (\%) was measured when the test mice were re-exposed to the context with or without their cagemate 2 days later. Two days later, freezing levels were measured when test mice were exposed to a novel anxiogenic environment (a brightly lit open field) with or without their cagemate. Groups were counterbalanced such that mice exposed to the FC chamber with a cagemate were introduced into the open field apparatus alone and vice versa (Fig. 1a).

To determine whether the presence of a familiar object also affected freezing, we repeated the above experiment. Experimental mice were housed in same-sex pairs to avoid confounds of social isolation. A novel object ( $50 \mathrm{ml}$ conical tube) was added to the cage $48 \mathrm{~h}$ after pair housing. Subsequent fear conditioning, context re-exposure, and introduction to a novel context were carried out as described above (Fig. 1f).

In a separate, independent experiment, we also measured freezing when mice were exposed to a lemon scent that was present during FC (Fig. 1k). For all behavioral tests, mice were habituated to the room at least $30 \mathrm{~min}$ before testing. Details of behavioral testing are provided in Supplementary Methods.

\section{IL-PFC c-fos levels following social interaction}

c-fos induction. Animals were transferred to a fresh individual cages and housed for $48 \mathrm{~h}$ with an ovariectomized female. To examine patterns of c-fos induction in response to novel or familiar mouse or a novel object, the ovariectomized female was removed from the cage for $1 \mathrm{~h}$ and then either a novel object (50 $\mathrm{ml}$ conical tube), novel ovariectomized female, or the ovariectomized cagemate was placed in the test animal's home cage. The stimulus animal/object was removed after $50 \mathrm{~min}$, and test mice were perfused and brain tissue was collected 60 min after initial stimulus introduction. Tissue was processed for c-fos via immunolabeling and $\mathrm{c}_{\text {-fos }}{ }^{+}$cells were counted within the IL-PFC (detailed in Supplementary Methods, Fig. 2a, b, and Supplementary Fig. S2).

Labeling social neural ensembles in the IL-PFC

ArcCreER $R^{T 2}$-mediated cell labeling. Recombination was induced with 4-hydroxytamoxifen (4-OHT, Sigma-Aldrich) [51]. 4-OHT was dissolved by sonication in $10 \% \mathrm{EtOH} / 90 \%$ corn oil at a concentration of $10 \mathrm{mg} / \mathrm{ml}$. All mice were singly housed for 2 days. On the morning of day 3 , mice received a single intraperitoneal injection of $0.15 \mathrm{ml} 4-\mathrm{OHT}$ or vehicle (VEH) $(10 \% \mathrm{EtOH} / 90 \%$ corn oil) (Fig. 2c) [47]. Five hours after injection, mice were introduced to and subsequently lived with an ovariectomized female or novel object. Cages were left undisturbed with the lights off for $60 \mathrm{~h}$ until the normal light-dark cycle was resumed (Fig. 2d). Experimental mice consisted of ArcCreER ${ }^{T 2}:$ Rosa $^{C h R 2-e Y F P}$ that received 4-OHT. Controls were ArcCreER ${ }^{\text {T2 }}::$ Rosa $^{\text {ChR2-eYFP }}$ that received VEH or Rosa ${ }^{C h R 2-e Y F P}, A^{2} C r e E R^{T 2}$, or wild-type littermates mice that received 4-OHT. Measurement of eYFP induction is detailed in Supplementary Methods.

Optogenetic manipulation of social neural ensembles ArcCreER ${ }^{T 2}$-mediated cell labeling. Social ensembles were labeled as described above, but all mice (genotypes for experimental and 

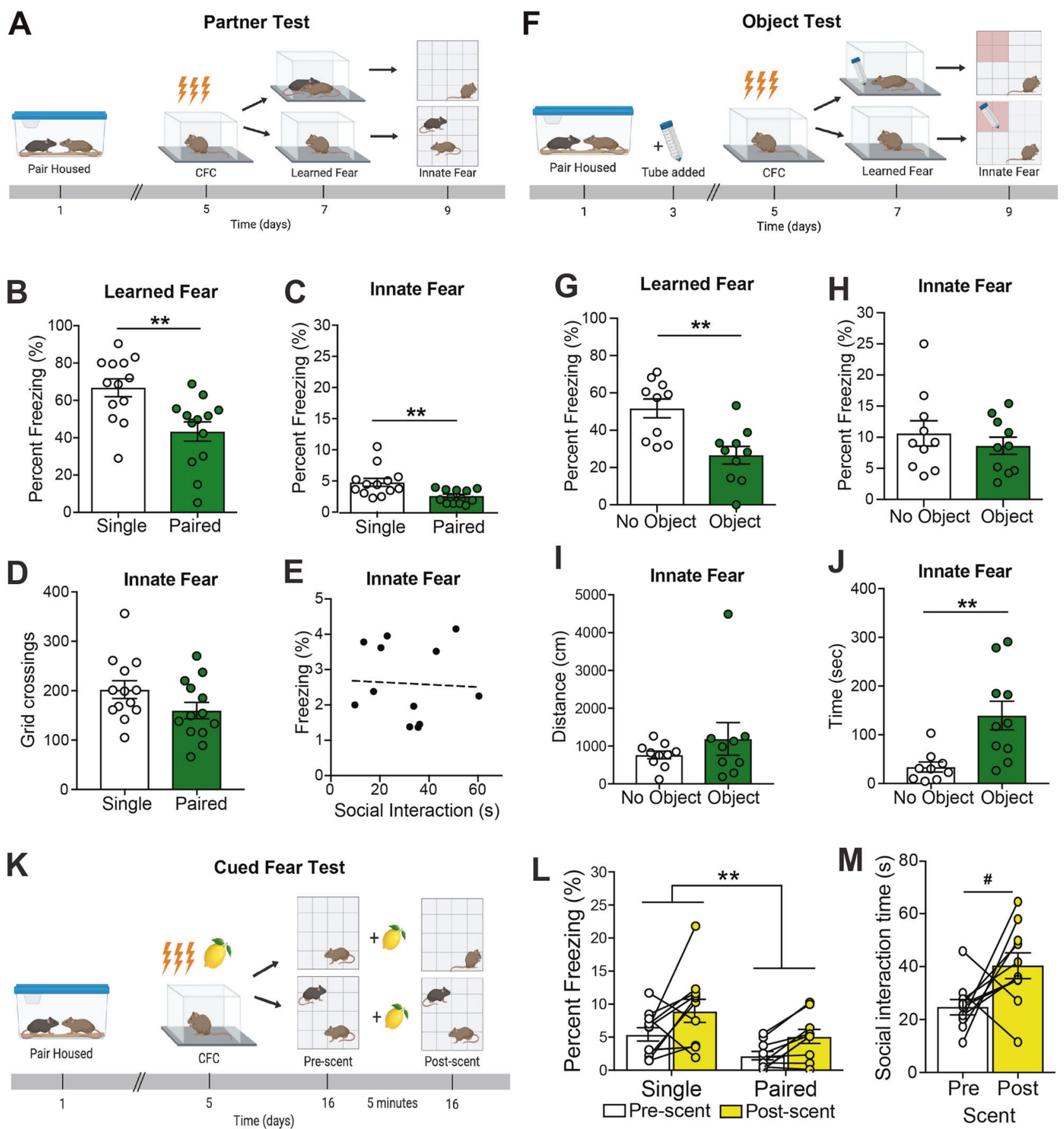

Fig. 1 Cagemate presence reduces freezing in multiple fear-provoking tests. a Timeline and behavioral schematic of testing with or without a cagemate. Learned fear was assessed by measuring freezing upon re-exposure to a context previously paired with an aversive shock, whereas innate fear was assessed in an anxiogenic novel environment. b Mice re-exposed to an aversive context with their cagemate showed lower levels of freezing when compared with mice tested alone. c Similar to learned fear, mice froze less in an innate fear context when tested with their cagemate present compared with mice tested alone. $\mathbf{d}$ Mice with and without their cagemate show similar locomotive behavior in the innate fear context. e There is no correlation between time investigating the cagemate and time freezing in the innate context for paired mice. f Timeline and behavioral schematic of testing with or without an object. g Mice re-exposed to an aversive context with a novel object showed lower levels of freezing when compared to mice tested alone. $\mathbf{h}$ Mice show similar freezing in an innate fear context when tested with a novel object present compared with mice tested alone. i Mice with and without an object show similar locomotive behavior in the innate fear context. $\mathbf{j}$ Mice spent more time in the object zone if the object was present. $\mathbf{k}$ Timeline and behavioral schematic showing aversive cue exposure with (bottom) or without (top) a cagemate present. After receiving a shock with a lemon scent present, mice were exposed to a novel context for $5 \mathrm{~min}$ and then the lemon scent (cue) was added to the arena for another 5 min. The lemon scent was the only shock-associated cue present in the test. I All mice increased their freezing levels after presentation of the lemon scent, but cagemate presence reduce freezing before and after scent presentation relative to mice tested alone. $\mathbf{m}$ Paired mice showed a trend towards increased interaction with their cagemate after the scent (fear cue) was added to the chamber. $n=10-13$ mice per group. Error bars represent \pm SEM. ${ }^{*} p<0.05 ;{ }^{* *} p<0.01,{ }^{* *} p<0.001, \# p=0.055$ CFC, contextual fear conditioning. 
A

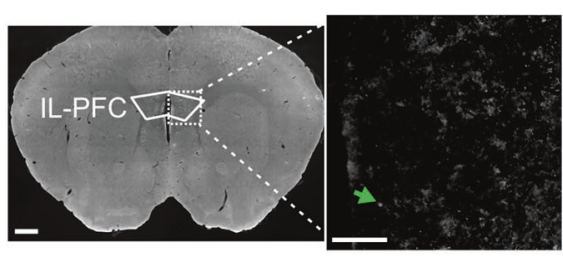

Control

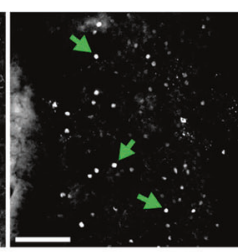

Novel

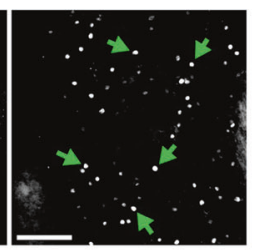

Familiar
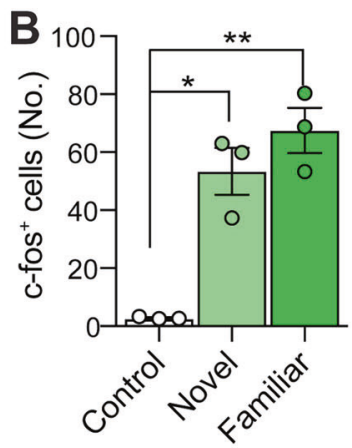

C

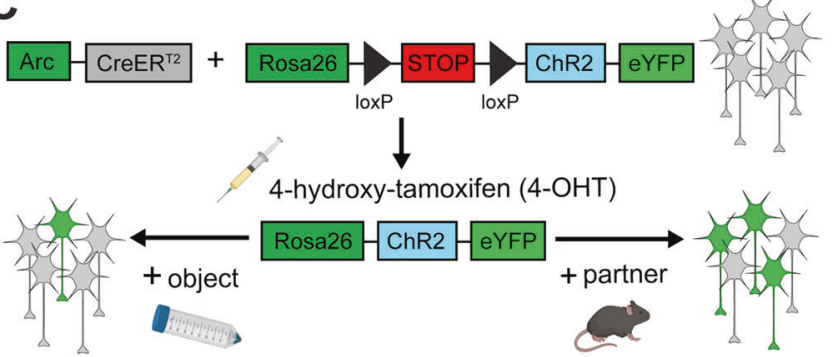

D

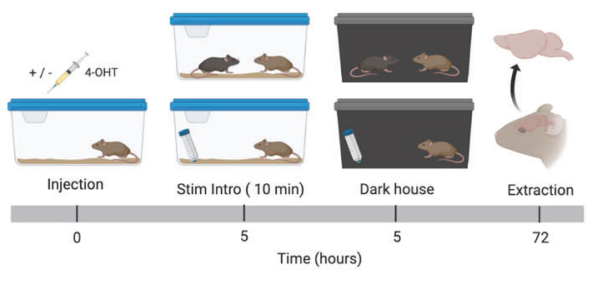

E

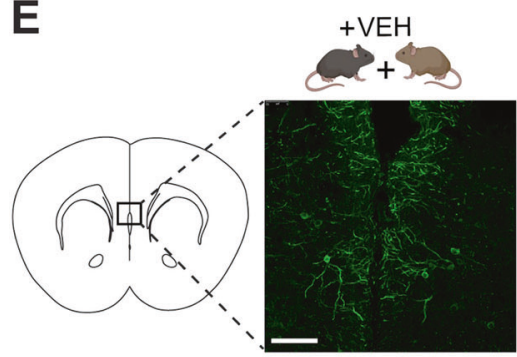

Control

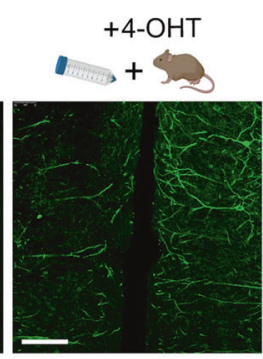

Object

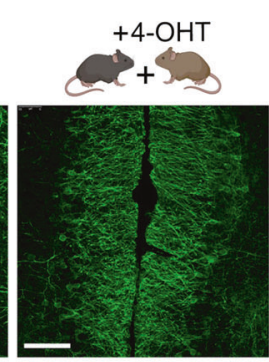

Partner

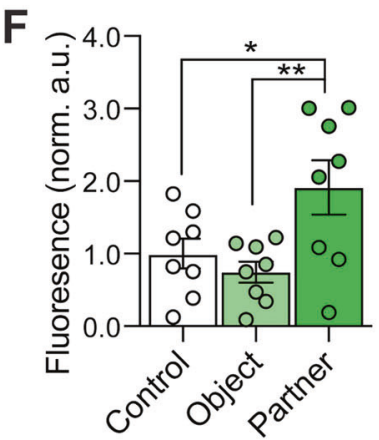

Fig. 2 The IL-PFC exhibits robust IEG induction and IEG-mediated cellular tagging following social interaction. a Representative images of c-fos induction following exposure to a novel or familiar mouse, compared with controls exposed to a novel object (conical tube). Scale bars left $=10 \mathrm{~mm}$, right $=50 \mu \mathrm{m}$. b Quantification of c-fos ${ }^{+}$cells in mice exposed to a novel object $(n=3)$, a novel mouse $(n=6)$, or a familiar mouse $(n=4)$. Exposure to a novel or familiar mouse results in significant induction of c-fos ${ }^{+}$cells relative to object-exposed controls, but there were no differences between socially exposed groups $(p=0.098)$. c ArcCreER ${ }^{\text {T2 }} \times$ Rosa $^{\text {ChR2-eYFP }}$ mice were used to indelibly label socially active neurons with ChR2-eYFP. Briefly, CreER ${ }^{12}$ is under the control of the Arc promoter. Upon administration of 4-OHT, CreER ${ }^{\mathrm{T} 2}$ localizes to the nucleus and recombines loxP sites in the floxed-STOP-ChR2-eYFP transgene, resulting in indelible expression of ChR2-eYFP in Arc ${ }^{+}$cells. Labeling ends when 4-OHT is metabolized and excreted. $\mathbf{d}$ Timeline for labeling a social neural ensemble in the IL-PFC. Mice received 4-OHT or vehicle and $5 \mathrm{~h}$ later were exposed to a new cagemate or novel object. e Representative images of immunolabeling of ChR2-eYFP ${ }^{+}$cells in the IL-PFC in mice receiving 4-OHT or vehicle prior to exposure to a novel mouse or a novel object. Scale bars $=50 \mu \mathrm{m}$. $\mathbf{f}$ Quantification of eYFP in the IL-PFC, where robust labeling was observed in mice receiving 4-OHT and exposed to a novel mouse compared with an object or vehicle controls ( $n=8$ mice per group). Error bars represent \pm SEM. ${ }^{*} p<0.05 ;{ }^{* *} p<0.01,{ }^{* * *} p<0.001$. Veh, vehicle; $4-\mathrm{OHT}$, 4 -hydroxytamoxifen; stim, stimulus; sac, sacrifice; norm, normalized.

control are outlined above) were paired with ovariectomized female $5 \mathrm{~h}$ after VEH or 4-OHT administration (Fig. 3a). Experimental mice continued to live with this female through the duration of the experiment. Behavioral testing was initiated 10-14 days after the VEH or 4-OHT injection, to ensure robust expression of ChR2-eYFP.

Optogenetics manipulation. Details regarding construction of optical fibers and their implantation are detailed in Supplementary Methods. For all optogenetic experiments, the laser was turned on at least $30 \mathrm{~min}$ before and tested for consistent light output prior to experimentation. The fiber optic implant was cleaned with an IPA wipe (VWR International) prior to attaching the patch cable. Output was normalized by individual implant for each mouse to $10-12 \mathrm{~mW}$. A master8 or custom Arduino interface was used to control light delivery, achieving $5 \mathrm{~ms}$ pulses at $10 \mathrm{~Hz}$.

Behavioral testing. For all behavioral experiments assessing the effects of activation of the social ensemble, mice lived with their cagemate but were tested alone. Experimental mice underwent three tests in the following order: conditioned fear assessment (learned fear), exposure to a novel environment (innate fear), and real-time place preference (RTPP) (Fig. 3a). Details available in Supplementary Methods.

Data analysis

All data are presented as mean \pm SEM. Data analysis was conducted using Statistical Package for the Social Sciences version 24 IBM software. Specific statistical tests used are indicated above 

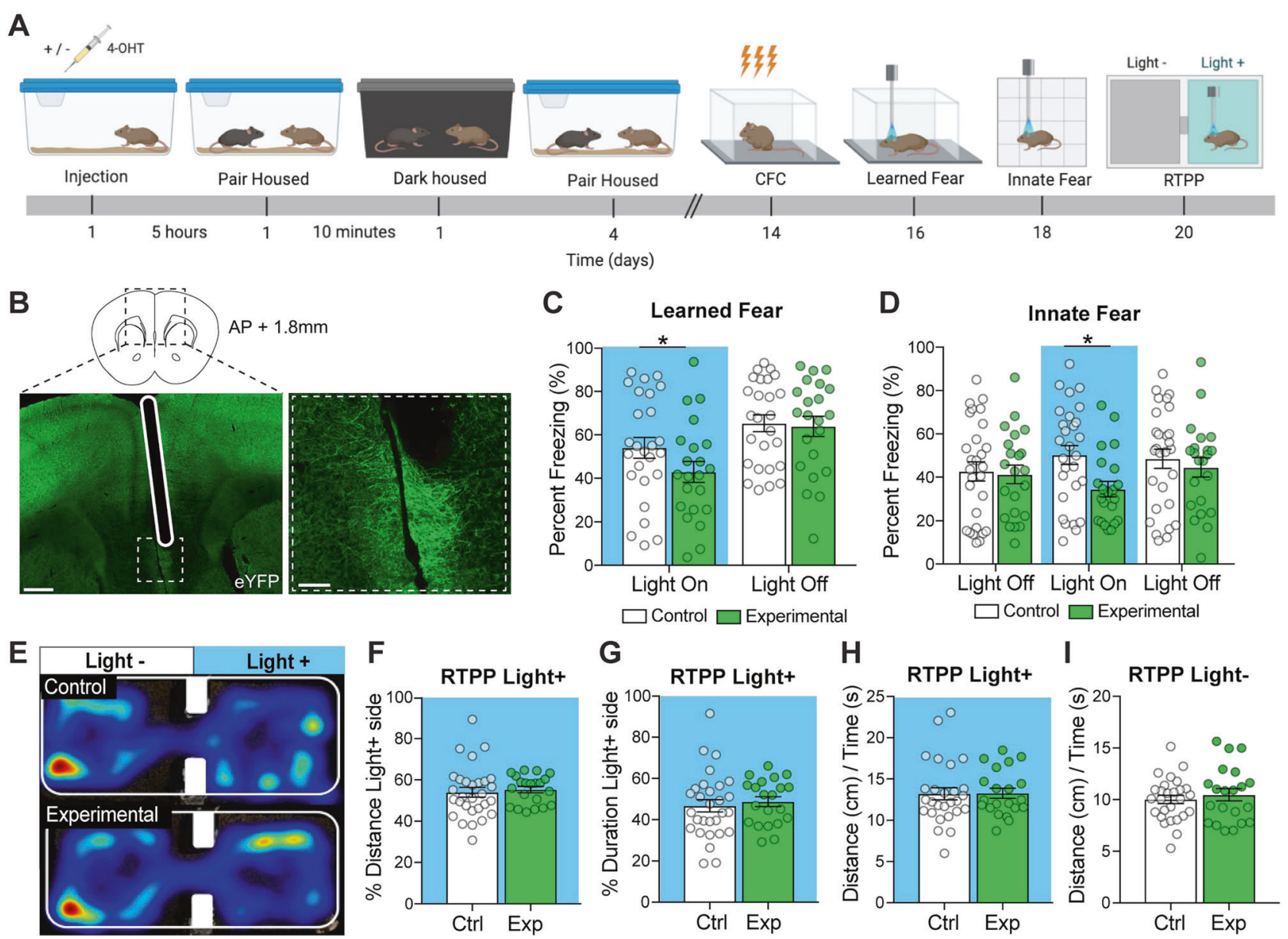

Fig. 3 Optogenetic reactivation of social neural ensembles reduces freezing in multiple tests. a Behavioral timeline for optogenetic cohorts. $\mathbf{b}$ Visualization of fiber optic implanted in the midline of the IL-PFC (below) and magnified image of eYFP+ socially labeled cells in the IL-PFC (right). Scale bars left $=100 \mu \mathrm{m}$, right $=50 \mu \mathrm{m}$. c Optogenetic reactivation of the socially labeled cells in the IL-PFC caused a reduction in freezing upon re-exposure to an environment previously paired with a shock. d Optogenetic reactivation of socially labeled ensembles significantly reduced freezing in a novel context during light-on period. e Representative heat maps for locomotion during RTPP (top $=$ control, bottom = experimental). Optogenetic stimulation of socially labeled cells in the IL-PFC was not aversive or rewarding. Total distance traveled in the light-on side divided by time spent in the light-on chamber $\mathbf{f}$ and the distance by time in the light-off chamber $\mathbf{g}$ was not significantly different between control and experimental mice. The percent distance traveled $\mathbf{h}$ and the duration $\mathbf{i}$ in the light-on chamber was not significantly different between control and experimental mice. $n=21-28$ mice per group, details in supplementary statistics table. Error bars represent \pm SEM. ${ }^{*} p<0.05 ;{ }^{* *} p<0.01,{ }^{* * *} p<0.001$. 4-OHT, 4-hydroxytamoxifen; CFC, contextual fear conditioning; RTPP, real-time place preference; blue, light ON.

and detailed results of statistical analyses and group sizes are available in Supplementary Table 1.

\section{RESULTS}

Social buffering occurs in multiple fear-provoking contexts We developed methods to assess social buffering across a range of tests that varied in the extent to which they elicited freezing. In the most intense of these tests, mice were re-exposed to a context in which they had previously received aversive shocks (Fig. 1a). Mice that were placed in this context with their cagemate exhibited lower levels of freezing than mice that were placed in this context alone (Fig. 1b). Specifically, Cagemate Presence had a significant main effect on freezing levels $(p<0.001)$, as did Sex of the experimental animal ( $p=0.007$; Supplementary Fig. S1a, b), but there was no interaction between Cagemate Presence and Sex $(p=0.294)$. We also exposed the same subjects to a separate novel context. Mice who were introduced with their cagemate present exhibited decreased freezing compared with the mice placed in the apparatus alone (Fig. 1c). Specifically, Cagemate
Presence had a significant main effect on freezing levels ( $p=$ $0.008)$, but there was no effect of Sex of the experimental animal $(p=0.504)$ or interaction between Cagemate Presence and Sex $(p=0.775$ ) (Supplementary Fig. S1a, b). These two tests produced freezing levels that differed by an order of magnitude, suggesting substantial differences in the amount of fear elicited by each test. Cagemate presence had a significant effect on freezing levels in both tests, suggesting that social buffering effects are evident across a wide range of fear-provoking/anxiogenic environments.

To test whether a familiar object elicited a similar reduction in freezing, we conducted the same experiment with a conical tube from the animal's home cage (Fig. 1f). Mice placed in the FC chamber with the familiar object froze less than their counterparts re-exposed without an object (Fig. 1g). Specifically, Object Presence had a significant main effect on freezing levels ( $p=$ $0.005)$, but there was no effect of Sex of the experimental animal $(p=0.93)$ or interaction between Cagemate Presence and Sex $(p=0.86)$ (Supplementary Fig. S1e, f). Next, we exposed the mice to a novel anxiogenic context; mice that had been exposed to the fear-conditioning chamber alone were exposed to the novel 
context with the object and vice versa. We found that the presence of a familiar object had no effect on freezing in the novel anxiogenic environment (Fig. $1 \mathrm{~h}$; main effect of Object, $p=0.51$, Object $\times$ Sex,$p=0.73$ ) despite the fact that mice spent more time near the object if it was present in the chamber (Fig. 1j, $p=0.005$ ). Overall, the levels of freezing were higher in the object test than in the social test, which may be attributable to some generalization as the same experimenter performed the learned and innate tasks. Thus, even though the mice prefer to be near the familiar object, its effect on freezing does not appear to generalize across learned and innately fear-provoking environments, at least within the context of these two specific behavioral tests.

We next asked whether differences in locomotion might be sufficient to explain socially mediated differences in freezing. We focused on behavior in the novel environment, which due to its size, enables more locomotion than the fear-conditioning chamber. In the novel environment, we observed that only social and not object presence reduced freezing. We furthermore found that neither the presence of a cagemate nor the object altered locomotion (Fig. 1d, h; social $p=0.096$ with less locomotion in paired animals; object $p=0.33$ ). Thus, gross locomotion (due to, e.g., chasing) does not explain social buffering of freezing. We next asked whether social interaction itself was responsible for the reduction in freezing by examining the correlation between freezing and social interaction initiated by the test animal. These two metrics were not correlated (Fig. 1e; $r=-0.061, p=0.84$ ), suggesting that physical interaction was also not the primary driver of reduced freezing.

In a separate cohort, we also examined the role of cagemate presence on cued olfactory fear responses. After being trained to associate a lemon scent with an aversive shock, the test animal was placed in a novel context with or without their cagemate. A comparison of freezing levels before (pre-scent) and after (postscent) re-exposure to the lemon scent (cue) (Fig. 1k) revealed an increase in freezing following scent introduction (Fig. 1l, $p=$ 0.005). However, freezing levels were significantly decreased both pre- and post-scent if the animal was placed in the context with their cagemate (Fig. 1l, $p=0.019$ ). When separated by sex, this social buffering effect was significant in males (Supplementary Fig. S1m, $p=0.011$ ) but not in females (Supplementary Fig. S1n, $p=0.58$ ). However, this may reflect the small number of female mice available for this particular test. In this test, freezing levels before cue introduction were comparable to those observed in the novel environment and increased to an intermediate level following scent application. Finally, among mice that had their cagemate present during testing, the time spent interacting with the cagemate tended to increase following scent addition (Fig. 1m, $p=0.055)$.

Taken together, these results indicate that having a cagemate present blunts freezing in a range of fear-provoking situations, providing experimental evidence that cagemate presence produces a robust social buffering effect evident at a behavioral level. In addition, both males and females are sensitive to cagemate presence, although to a different extent in different tests.

IL-PFC exhibits robust induction of c-fos following social but not object interaction

Having demonstrated that cagemate presence decreases a behavioral metric of fear, and based on the known role of the IL-PFC in fear modulation, we next asked whether IEG expression was upregulated in the IL-PFC following social interaction. Specifically, we compared c-fos expression in the IL-PFC in mice exposed to a familiar ovariectomized female (familiar), novel ovariectomized female (novel), or a novel object (control) (Fig. 2a and Supplementary Fig. S2). We found that there were significantly more $c$-fos ${ }^{+}$cells in the IL-PFC of mice exposed to a novel $(p=0.030)$ or familiar $(p=0.003)$ mouse as compared with controls (Fisher's multiple comparisons test). There was no difference in number of $\mathrm{c}^{-} \mathrm{fos}^{+}$cells found between the novel and familiar exposure conditions (Fig. 2b, $p=0.097$ ). Thus, these data indicate that social interaction substantially increases IEG expression in the IL-PFC.

Optogenetic activation of social neural ensembles in IL-PFC reduces freezing

In order to identify and manipulate social ensembles, we used the ArcCreER ${ }^{T 2} \times$ Ai32 mice to express ChR2-eYFP in socially active neurons (Fig. 2c, d). In contrast to sparsely labeled areas, such as the dentate gyrus [52], dense labeling with membrane-bound eYFP in the IL-PFC made identification of individual soma and full colocalization of a somatic IEG untenable. We observed a greater amount of eYFP expression in the IL-PFC of mice paired with an ovariectomized female as compared with controls (Fig. 2e, $f$ and Supplementary Fig. S2, $p=0.012$ ), mirroring socially induced IEG expression (Fig. 2b).

To test whether activation of the social neural ensemble in the IL-PFC modulates freezing levels, we optogenetically stimulated

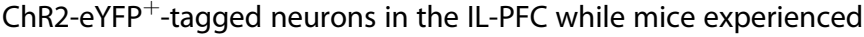
different fear-provoking environments. The experimental timeline is summarized in Fig. 3a. Light-induced activation of the social ensemble reduced freezing when mice were placed in a context in which they had previously been shocked (Fig. $3 c$ and Supplementary Fig. S3a, Light $\times$ Group: $p=0.036$ ). In addition, experimental mice exhibited a greater increase in freezing following the light being turned off as compared with controls $(p=0.040$; Fig. 3c). Light-induced activation of the same ensemble also reduced freezing in a novel arena, which returned to control levels when the laser was turned off (Fig. 3d and Supplementary Fig. $\mathrm{S} 3 \mathrm{~b}$; Light $\times$ Group: $p=0.010$ ). The effects of ensemble activation were strongest immediately following light onset; control mice did not change their freezing $(p=0.64)$, but experimental mice decreased their freezing by nearly $20 \%(p=0.005)$ (Supplementary Fig. S3b). Notably, freezing levels in the novel environment were greater in our optogenetic experiments than in prior behavioral tests (e.g., Fig. 1c). All mice had the fiber optic attached to the ferrule during FC. Thus, the fiber optic cable may have served as an aversive cue and contributed to the elevated freezing levels in the novel context. For all analyses, males and females were combined as there was no main effect or interacting effects of Sex. This indicates that reactivation of socially active neurons in the IL-PFC mimics the effects of social buffering.

One concern regarding the above finding is that the $\mathrm{ArcCreER} \mathrm{R}^{\mathrm{T2}}$ mouse line exhibits brain region-specific levels of "leak"; labeled cells are observed even in the absence of 4-OHT administration, because Cre-recombinase can escape sequestration in the cytoplasm (Fig. 2e) [51]. To determine whether optogenetic reactivation of nonspecifically labeled (i.e., "leak") cells was sufficient to reduce freezing, we limited our controls to VEHtreated ArcCreER ${ }^{T 2}::$ Rosa ${ }^{C h R 2-e Y F P}$ mice (leak controls) and compared their behavior with that of $4-\mathrm{OHT}$-treated $\operatorname{ArcCreER}^{\mathrm{T2}}$ :: Rosa ${ }^{\text {ChR2-eYFP }}$ mice (experimental). We found that light application did not reduce freezing in leak controls (Supplementary Fig. S3c, learned fear: light-induced change in freezing in leak vs experimental, $p=0.00029$; Supplementary Fig. S3d, innate fear: no light-mediated onset of freezing in control group, $p=0.87$ vs. light-mediated onset of freezing in experimental, $p=0.005$ ). This indicates that nonspecific labeling is not sufficient to produce the anxiolytic effects attributable to optogenetic activation of the social neural ensemble.

The transgenic strategy we used is notable for being able to capture, tag, and manipulate cells based on their activational attributes-in this case Arc expression upon cagemate introduction. This system relies on dual transgenes and optogenetic manipulation, both of which require appropriate controls. We controlled for transgene presence and nonspecific ChR2 expres-

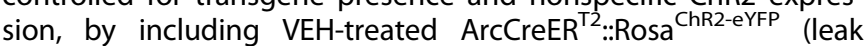


control) and 4-OHT-treated ArcCreER ${ }^{\mathrm{T2}}$ or Rosa ${ }^{\text {ChR2-eYFP }}$ mice (transgene controls). We controlled for light application using a within-subject design, examining behavior during light-ON and light-OFF periods. Freezing was attenuated exclusively in experimental mice during light $\mathrm{ON}$ periods in fear-provoking environments. Thus, combined with prior validation of the $\operatorname{ArcCreER}^{T 2}$ line [49, 51-54], we felt that inclusion of an eYFP-only control (e.g., 4-OHT-treated ArcCreER ${ }^{\mathrm{T2}}: \mathrm{Rosa}^{\mathrm{eYFP}}$ ) was not necessary.

Reactivation of social neural ensembles is not rewarding or aversive and does not alter locomotion

Using a RTPP test, we found that stimulation of the social neural ensemble was not inherently aversive or rewarding and did not affect locomotion. Specifically, stimulation of the ensemble did not alter the percent locomotion when the mice were in the light ON chamber compared with the light OFF chamber (Fig. 3f, Supplementary Fig. S3e, $p=0.62$ ). Similarly, there was no difference in the amount of time that the mice spent in the light ON chamber compared with the light OFF chamber (Fig. $3 \mathrm{~g}$ and Supplementary Fig. S3f, $p=0.68$ ) and no difference in locomotion rate $(\mathrm{cm} / \mathrm{s})$ between experimental and control mice in either chamber (Fig. 3h-i and Supplementary Fig. S3g-h, light ON $p=$ 0.61 , light OFF $p=0.28$ ). For all analyses, males and females were combined, as there was no main or interacting effect of Sex. Thus, the differences in freezing observed during optogenetic manipulation are not attributable to acute induction of locomotor or reward-related processes.

\section{DISCUSSION}

The buffering of fear responses by conspecific presence is a broadly observed and highly conserved phenomenon [29, 55]. We have demonstrated that companion presence can attenuate freezing in mice, and that this effect can be recapitulated in the absence of a conspecific by optogenetically reactivating socially active neurons in the IL-PFC. Further, we demonstrated that this effect is evident across fear-provoking situations that vary in intensity, although additional studies are needed to determine the full extent of generalization. This suggests that social neural ensembles within the IL-PFC may exert top-down control to modulate the behavioral expression of fear, and that this is not necessarily context- or cue-specific.

In order to gain insight into whether familiarity alone is sufficient to provide our observed anxiolytic effects, we tested whether the presence of a familiar object also attenuated freezing. We found that the presence of a familiar object reduced freezing upon re-exposure to an aversive context previously paired with a shock but not in a novel anxiogenic environment. One potential explanation for these findings is that the object alters the perception of the fear-conditioning context. Previous studies have shown that changes to the context are sufficient to decrease freezing $[56,57]$. The lack of effect in a novel environment is consistent with the innate nature of this task; freezing does not depend on a previously learned aversive contextual association. Together, these results suggest that freezing in learned fear contexts is sensitive to a variety of social and non-social factors, and that cagemate presence is more anxiolytic than the presence of a familiar object in innately anxiogenic environments.

We also examined whether decreased freezing was attributable to differences in locomotion. The presence of a cagemate or an object did not alter locomotion in a novel environment, and light application did not alter locomotion in our optogenetic experiment. In addition, the amount of social interaction initiated by the test animal in the novel arena was not correlated with freezing. Together, this suggests that the presence of a familiar, unafraid conspecific is sufficient to reduce freezing independent of locomotor activity. The cagemate may act as a safety cue, as would be suggested by studies in which previous positive social associations were enough to buffer freezing in a learned aversive context [58]. The IL-PFC is also required for appropriate discrimination of safety cues, suggesting that our socially labeled PFC neurons may contribute to the assessment of the cagemate as a safety signal [59].

Multiple lines of evidence also indicate that the IL-PFC processes social information and modulates fear responses, although few studies have examined the potential convergence of these functions. The IL-PFC exhibits robust neuronal activity during social interaction [30-32] and we confirmed that interaction with either a novel or familiar conspecific results in increased $\mathrm{c}^{-} \mathrm{fos}^{+}$expression within this region [60]. Likewise, the IL-PFC is required for extinction of fear [44-46]. Some studies directly or indirectly suggest a role for this region in social buffering. Specifically, inhibition of the IL-PFC via muscimol infusion acutely blocks the effects of social familiarity in reducing anxiety-like behaviors [38] and lesions of this region abolish the resiliencypromoting effects of environmental enrichment [61, 62]. The work presented here suggests that a specific subset of socially active neurons, potentially constituting a social memory trace, may mediate social buffering, further contributing to our understanding of how social processing and fear learning may be integrated within this brain region.

The prelimbic (PL)- and IL-PFC may be ideally suited to integrate multiple information streams. For instance, recent work revealed a specialized subset of social place cells in the PL-PFC that integrate social and spatial information [31]. Social neural ensembles in the IL-PFC may similarly integrate social memory and fear learning (or safety cues). But how does this fit into a distributed social memory circuit? Rodents rely on olfactory information to recognize other individuals and olfactory inputs are required for social buffering in rats $[11,63]$. Olfactory-based identity information eventually contributes to social memory engram in the ventral CA1 (vCA1) region of the HPC [64] and projections from this region to the PFC are required for social recognition memory $[42,65]$. Thus, social neural ensembles in the IL-PFC may represent an advanced level of social information processing reliant on social engram projections from vCA1 to the mPFC.

One novel aspect of our study is that it suggests that harnessing neurons activated by positive social interactions may be useful in broadly ameliorating fear and/or anxiety. Prior studies have captured a memory trace linked to a specific fearprovoking event/context $[49,66,67]$. Subsequent manipulation of these engrams has revealed essential aspects of memory and its plasticity $[53,68-71]$. Although this may have therapeutic potential for disorders linked to a specific traumatic event, such as PTSD, it provides less promise for addressing generalized anxiety disorder (GAD). In contrast, we observed reduced freezing levels across contextual, cued, and innately fearprovoking tasks if a cagemate was present, and our optogenetic effects were also evident across tasks. In the latter instance, activation of the social ensemble in the novel anxiogenic environment may represent blended innate and cued fear due to "carry over" from having the fiber optic cable attached during fear conditioning and being handled by the same experimenter. Thus, activation of IL-PFC social neural ensembles may be more broadly effective for modulating fear and anxiety, which may be relevant for treating GAD.

Social buffering encompasses a broad range of physiological and behavioral responses [55]. Here we focused exclusively on the modulation of freezing as a behavioral indication of fear. Previous work has suggested that the physiological and behavioral effects of social buffering may be dissociable; different social exposures can elicit different buffering responses. For instance, in rats, conspecific presence while experiencing an anxiogenic environment reduces freezing, whereas co-housing with a conspecific following fear conditioning during the recovery period attenuates autonomic responses (specifically hypothermia) without altering 
freezing [28, 72]. Other studies have also examined how hypothalamo-pituitary-adrenal axis activity is modulated by conspecific presence [73-75]. Determining whether activation of social neural ensembles in the IL-PFC can ameliorate stress responses, in addition to fear, will provide insight into the neural architecture of different facets of social buffering. Likewise, this will be critical for determining potential therapeutic opportunities. For instance, if activation of social neural ensembles ameliorates the affective changes attributable to chronic stress exposure, this would potentially be promising for addressing depressionrelated behaviors.

Although we found that optogenetic reactivation of socially labeled neurons attenuated fear, there remain a number of questions regarding the identity of these neurons and how they are recruited by social interaction. In particular, social interactions are complex and the labeling window in ArcCreER ${ }^{\mathrm{T2}}$ mice is sufficiently long that a variety of factors related to social reward, sensory processing, memory, and exploratory behavior could all contribute to the labeling of these cells. Many of these processes, such as reward, learning, and exploration of novelty, are not limited to social behavior and thus non-social information may also contribute to the labeling of these cells. To tease this apart, future work should focus on identifying the specific factors that activate these cells by visualizing and functionally interrogating cells labeled following exposure to non-social novelty, reward, discrete sensory stimuli (such as urine cues), or randomly labeled cells (e.g., via low-titer viral expression of ChR2). Such comparisons require significant effort but will parse cell-identity-specific effects from those that perhaps scale with the number of randomly labeled cells.

Another question that remains is how social ensembles regulate fear responses within a larger neural circuit. Previous work has shown that the mPFC exerts top-down control of fear and anxiety [76]. Specifically, projections from the IL-PFC to the basolateral amygdala (BLA) are required for fear extinction $[34,44]$. If social interaction preferentially recruits IL-BLAprojecting neurons, this could explain how activation of these cells regulates fear expression. In addition, oxytocin is required for social buffering and oxytocin signaling in the PL cortex reduces anxiety $[10,14,22,58,77]$. One intriguing possibility is that oxytocin signaling either directly or via connections from the mPFC recruits the subset of cells in the IL-PFC that are active during social interaction.

In addition to the areas of further investigation outlined above, our approach also has a few limitations. In particular, we focused on freezing as a metric of fear expression, but we did not limit interaction between the test animal and their cagemate during testing. Thus, freezing could also be reduced because of the cagemate physically investigating the test animal. These concerns are mitigated because optogenetic activation, where only the test animal was present, resulted in similar decreases in freezing. However, as with all manipulations that measure freezing, it is possible that our interventions did not directly ameliorate fear but instead distracted the subject from a fearful context and/or engaged an exploratory drive. In addition, the scope of our work is limited to gain-of-function optogenetic manipulations, and as such, we cannot make any conclusions about the necessity of socially active IL-PFC neurons for the effects of social buffering. One potential way to address the requirement of these neurons for social buffering would be to optogenetically inactivate these cells while mice are exposed to fear-provoking environments with a cagemate present. Finally, we tested the effects of only a single type of social stimulus, ovariectomized female cagemates, and it is possible that different social partners could engage different neuronal ensembles, thereby producing different effects on fear levels. The latter has been well documented in rats and to some extent in mice and voles, where the developmental stage, emotional state, and affiliative nature of the companion animal are all important modulators of social buffering [58, 78-81].

In summary, social buffering is a powerful regulator of fear and anxiety across species, and our work provides a potential cellular substrate that mediates the effects of companion presence on fear expression. Despite differences in the sensory factors that are important for social buffering in rodents (olfactory/tactile) and humans (visual/tactile), substantial evidence suggests that similar socially sensitive neural circuits are recruited to reduce fear responses in humans and rodents [29]. Within this circuitry, the PFC is ideally positioned to integrate sensory information and exert top-down social regulation of emotional states [33, 82]. As such, understanding the neural dynamics and circuits that underlie social buffering may contribute to novel therapeutics for fear and anxiety disorders.

\section{FUNDING AND DISCLOSURE}

This work was supported by R00 MH102352, DP2OD026143, and a Young Investigator Grant from the American Foundation for Suicide Prevention (Z.R.D.). C.A.D. is named on patent applications for the prophylactic use of $(R, S)$-ketamine and other drugs against stress-related psychiatric disorders. All other authors declare no conflict of interest.

\section{ACKNOWLEDGEMENTS}

We thank the animal care staff at CUIMC and University of Colorado Boulder and the staff of the CU Boulder MCDB Light Microscopy Facility. Additional technical assistance was provided by Magdalena Woroniecka, Kelsey Harbert, and Katelyn Gordon.

\section{AUTHOR CONTRIBUTIONS}

Z.R.D. and C.A.D. designed research. V.A.G., K.A., T.L.S., A.M.C., M.S., and A.M.Z. performed research. V.A.G., K.A., M.S., and A.M.Z. analyzed data. V.A.G. and Z.R.D. wrote the paper. This article contains supporting information online.

\section{ADDITIONAL INFORMATION}

Supplementary Information accompanies this paper at (https://doi.org/10.1038/ s41386-020-0631-1).

Publisher's note Springer Nature remains neutral with regard to jurisdictional claims in published maps and institutional affiliations.

\section{REFERENCES}

1. DeVries AC, Glasper ER, Detillion CE. Social modulation of stress responses. Physiol Behav. 2003;79:399-407.

2. Holt-Lunstad J, Smith TB, Layton JB. Social relationships and mortality risk: a meta-analytic review. PLOS Med. 2010;7:e1000316.

3. House JS, Landis KR, Umberson D. Social relationships and health. Science 1988:241:540-5.

4. Ozbay F, Fitterling H, Charney D, Southwick S. Social support and resilience to stress across the life span: a neurobiologic framework. Curr Psychiatry Rep. 2008;10:304-10.

5. Ozbay F, Johnson DC, Dimoulas E, Morgan CA, Charney D, Southwick S. Social support and resilience to stress: from neurobiology to clinical practice. Psychiatry (Edgmont) 2007;4:35-40.

6. Reblin M, Uchino BN. Social and emotional support and its implication for health Curr Opin Psychiatry 2008;21:201-5.

7. Rodríguez-Artalejo F, Guallar-Castillón P, Herrera MC, Otero CM, Chiva MO, Ochoa CC, et al. Social network as a predictor of hospital readmission and mortality among older patients with heart failure. J Card Fail. 2006;12:621-7.

8. Valtorta NK, Kanaan M, Gilbody S, Ronzi S, Hanratty B. Loneliness and social isolation as risk factors for coronary heart disease and stroke: systematic review and meta-analysis of longitudinal observational studies. Heart 2016;102:1009-16.

9. Archie EA, Tung J, Clark M, Altmann J, Alberts SC. Social affiliation matters: both same-sex and opposite-sex relationships predict survival in wild female baboons. Proc Biol Sci. 2014;281:pii: 20141261. 
10. Kikusui T, Winslow JT, Mori Y. Social buffering: relief from stress and anxiety. Philos Trans R Soc Lond Ser B Biol Sci. 2006;361:2215-28.

11. Kiyokawa $Y$, Wakabayashi $Y$, Takeuchi $Y$, Mori $Y$. The neural pathway underlying social buffering of conditioned fear responses in male rats. Eur J Neurosci. 2012;36:3429-37.

12. Beery AK, Kaufer D. Stress, social behavior, and resilience: Insights from rodents. Neurobiol Stress 2015;1:116-27.

13. Bovard EW. The effects of social stimuli on the response to stress. Psychol Rev. 1959;66:267-77.

14. Armario A, Luna G, Balasch J. The effect of conspecifics on corticoadrenal response of rats to a novel environment. Behav Neural Biol. 1983;37:332-7.

15. Davitz JR, Mason DJ. Socially facilitated reduction of a fear response in rats. J Comp Physiol Psychol. 1955;48:149-51.

16. Faustino Al, Tacão-Monteiro A, Oliveira RF. Mechanisms of social buffering of fear in zebrafish. Sci Rep. 2017:7:44329.

17. Lyons DM, Price EO, Moberg GP. Social grouping tendencies and separationinduced distress in juvenile sheep and goats. Dev Psychobiol. 1993;26:251-9.

18. Kanitz E, Hameister T, Tuchscherer M, Tuchscherer A, Puppe B. Social support attenuates the adverse consequences of social deprivation stress in domestic piglets. Horm Behav. 2014;65:203-10.

19. Galvão-Coelho NL, Silva HPA, De Sousa MBC. The influence of sex and relatedness on stress response in common marmosets (Callithrix jacchus). Am J Primatol. 2012;74:819-27.

20. Mendoza SP, Coe $\mathrm{CL}$, Lowe $\mathrm{EL}$, Levine $\mathrm{S}$. The physiological response to group formation in adult male squirrel monkeys. Psychoneuroendocrinology 1978;3:221-9.

21. Sanchez MM, McCormack KM, Howell BR. Social buffering of stress responses in nonhuman primates: maternal regulation of the development of emotional regulatory brain circuits. Soc Neurosci. 2015;10:512-26.

22. Winslow JT, Noble PL, Lyons CK, Sterk SM, Insel TR. Rearing effects on cerebrospinal fluid oxytocin concentration and social buffering in rhesus monkeys. Neuropsychopharmacology 2003;28:910-8.

23. Wittig RM, Crockford C, Weltring A, Langergraber KE, Deschner T, Zuberbühler $\mathrm{K}$. Social support reduces stress hormone levels in wild chimpanzees across stressful events and everyday affiliations. Nat Commun. 2016;7:13361.

24. Gunnar MR, Hostinar CE. The social buffering of the hypothalamic-pituitaryadrenocortical axis in humans: developmental and experiential determinants. Soc Neurosci. 2015;10:479-88.

25. Ditzen B, Heinrichs M. Psychobiology of social support: the social dimension of stress buffering. Restor Neurol Neurosci. 2014;32:149-62.

26. Stanton ME, Patterson JM, Levine S. Social influences on conditioned cortisol secretion in the squirrel monkey. Psychoneuroendocrinology 1985;10: 125-34

27. Kiyokawa Y, Hiroshima S, Takeuchi Y, Mori Y. Social buffering reduces male rats' behavioral and corticosterone responses to a conditioned stimulus. Horm Behav. 2014;65:114-8.

28. Kiyokawa $Y$, Takeuchi $Y$, Mori $Y$. Two types of social buffering differentially mitigate conditioned fear responses. Eur J Neurosci. 2007;26:3606-13.

29. Gunnar MR, Hostinar CE, Sanchez MM, Tottenham N, Sullivan RM. Parental buffering of fear and stress neurobiology: reviewing parallels across rodent, monkey, and human models. Soc Neurosci. 2015:10:474-8.

30. Kingsbury L, Huang S, Wang J, Gu K, Golshani P, Wu YE, et al. Correlated neural activity and encoding of behavior across brains of socially interacting animals. Cell 2019;178:429-.e16

31. Murugan M, Jang HJ, Park M, Miller EM, Cox J, Taliaferro JP, et al. Combined social and spatial coding in a descending projection from the prefrontal cortex. Cell 2017;171:1663-.e16.

32. Yizhar O, Fenno LE, Prigge M, Schneider F, Davidson TJ, O'Shea DJ, et al. Neocortical excitation/inhibition balance in information processing and social dysfunction. Nature 2011:477:171-8.

33. Gilmartin MR, Balderston NL, Helmstetter FJ. Prefrontal cortical regulation of fear learning. Trends Neurosci. 2014;37:455-64.

34. Orsini CA, Kim JH, Knapska E, Maren S. Hippocampal and prefrontal projections to the basal amygdala mediate contextual regulation of fear after extinction. J Neurosci. 2011;31:17269-77.

35. Feder A, Nestler EJ, Charney DS. Psychobiology and molecular genetics of resilience. Nat Rev Neurosci. 2009;10:446-57.

36. Maier SF, Watkins LR. Role of the medial prefrontal cortex in coping and resilience. Brain Res. 2010;1355:52-60.

37. Russo SJ, Murrough JW, Han M, Charney DS, Nestler EJ. Neurobiology of resilience. Nat Neurosci. 2012;15:1475-84.

38. Lungwitz EA, Stuber GD, Johnson PL, Dietrich AD, Schartz N, Hanrahan B, et al. The role of the medial prefrontal cortex in regulating social familiarity-induced anxiolysis. Neuropsychopharmacology 2014;39:1009-19.
39. Josselyn SA, Kohler S, Frankland PW. Finding the engram. Nat Rev Neurosci. 2015;16:521-34.

40. Denny CA, Lebois E, Ramirez S. From engrams to pathologies of the brain. Front Neural Circuits 2017:11:23.

41. Tonegawa S, Liu X, Ramirez S, Redondo R. Memory engram cells have come of age. Neuron 2015;87:918-31.

42. Okuyama T, Kitamura T, Roy DS, Itohara S, Tonegawa S. Ventral CA1 neurons store social memory. Science (NY, NY) 2016;353:1536-41.

43. Sakurai K, Zhao S, Takatoh J, Rodriguez E, Lu J, Leavitt AD, et al. Capturing and manipulating activated neuronal ensembles with CANE delineates a hypothalamic social-fear circuit. Neuron 2016;92:739-53.

44. Bloodgood DW, Sugam JA, Holmes A, Kash TL. Fear extinction requires infralimbic cortex projections to the basolateral amygdala. Transl Psychiatry 2018;8:60.

45. Barker JM, Taylor JR, Chandler LJ. A unifying model of the role of the infralimbic cortex in extinction and habits. Learn Mem. 2014;21:441-8.

46. Thompson BM, Baratta MV, Biedenkapp JC, Rudy JW, Watkins LR, Maier SF. Activation of the infralimbic cortex in a fear context enhances extinction learning. Learn Mem. 2010;17:591-9.

47. Colnaghi L, Clemenza K, Groleau SE, Weiss S, Snyder AM, Lopez-Rosas M, et al. Social involvement modulates the response to novel and adverse life events in mice. PLoS ONE 2016;11:e0163077.

48. Warren BL, Mendoza MP, Cruz FC, Leao RM, Caprioli D, Rubio FJ, et al. Distinct Fos-expressing neuronal ensembles in the ventromedial prefrontal cortex mediate food reward and extinction memories. J Neurosci. 2016;36: 6691-703.

49. Denny CA, Kheirbek MA, Alba EL, Tanaka KF, Brachman RA, Laughman KB, et al. Hippocampal memory traces are differentially modulated by experience, time, and adult neurogenesis. Neuron 2014;83:189-201.

50. Madisen L, Mao T, Koch H, Zhuo J, Berenyi A, Fujisawa S, et al. A toolbox of Credependent optogenetic transgenic mice for light-induced activation and silencing. Nat Neurosci. 2012;15:793-802.

51. Cazzulino AS, Martinez R, Tomm NK, Denny CA. Improved specificity of hippocampal memory trace labeling. Hippocampus 2016;26:752-62.

52. Perusini JN, Cajigas SA, Cohensedgh O, Lim SC, Pavlova IP, Donaldson ZR, et al. Optogenetic stimulation of dentate gyrus engrams restores memory in Alzheimer's disease mice. Hippocampus 2017;27:1110-22.

53. Lacagnina AF, Brockway ET, Crovetti CR, Shue F, McCarty MJ, Sattler KP, et al. Distinct hippocampal engrams control extinction and relapse of fear memory. Nat Neurosci. 2019;22:753-61.

54. Root CM, Denny CA, Hen R, Axel R. The participation of cortical amygdala in innate, odour-driven behaviour. Nature 2014;515:269-73.

55. Kiyokawa $Y$, Hennessy MB. Comparative studies of social buffering: a consideration of approaches, terminology, and pitfalls. Neurosci Biobehav Rev. 2018; 86:131-41.

56. Wotjak CT. Sound check, stage design and screen plot-how to increase the comparability of fear conditioning and fear extinction experiments. Psychopharmacology 2019;236:33-48.

57. Maren S, Phan KL, Liberzon I. The contextual brain: implications for fear conditioning, extinction and psychopathology. Nat Rev Neurosci. 2013; 14:417-28.

58. Guzman YF, Tronson NC, Sato K, Mesic I, Guedea AL, Nishimori K, et al. Role of oxytocin receptors in modulation of fear by social memory. Psychopharmacology (Berl). 2014;231:2097-105.

59. Sangha S, Robinson PD, Greba Q, Davies DA, Howland JG. Alterations in reward, fear and safety cue discrimination after Inactivation of the rat prelimbic and infralimbic cortices. Neuropsychopharmacology 2014;39:2405-13.

60. Kim Y, Venkataraju KU, Pradhan K, Mende C, Taranda J, Turaga SC, et al. Mapping social behavior-induced brain activation at cellular resolution in the mouse. Cell Rep. 2015;10:292-305.

61. Hinwood M, Tynan RJ, Day TA, Walker FR. Repeated social defeat selectively increases deltaFos $\mathrm{B}$ expression and histone $\mathrm{H} 3$ acetylation in the infralimbic medial prefrontal cortex. Cereb Cortex (NY, NY: 1991) 2011:21:262-71.

62. Lehmann ML, Herkenham M. Environmental enrichment confers stress resiliency to social defeat through an infralimbic cortex-dependent neuroanatomical pathway. J Neurosci. 2011;31:6159-73.

63. Matochik JA. Role of the main olfactory system in recognition between individual spiny mice. Physiol Behav. 1988;42:217-22.

64. Okuyama T. Social memory engram in the hippocampus. Neurosci Res. 2018;129:17-23.

65. Phillips ML, Robinson HA, Pozzo-Miller L. Ventral hippocampal projections to the medial prefrontal cortex regulate social memory. Elife 2019;8:pii: e44182.

66. Kitamura T, Sun C, Martin J, Kitch LJ, Schnitzer MJ, Tonegawa S. Entorhinal cortical ocean cells encode specific contexts and drive context-specific fear memory. Neuron 2015;87:1317-31 
67. Liu X, Ramirez S, Pang PT, Puryear CB, Govindarajan A, Deisseroth K, et al. Optogenetic stimulation of a hippocampal engram activates fear memory recall. Nature 2012;484:381-5.

68. Ramirez S, Liu X, MacDonald CJ, Moffa A, Zhou J, Redondo RL, et al. Activating positive memory engrams suppresses depression-like behaviour. Nature 2015; 522:335-9.

69. Redondo RL, Kim J, Arons AL, Ramirez S, Liu X, Tonegawa S. Bidirectional switch of the valence associated with a hippocampal contextual memory engram. Nature 2014;513:426-30.

70. Cai DJ, Aharoni D, Shuman T, Shobe J, Biane J, Song W, et al. A shared neural ensemble links distinct contextual memories encoded close in time. Nature 2016; 534:115-8.

71. Chen BK, Murawski NJ, Cincotta C, McKissick O, Finkelstein A, Hamidi AB, et al. Artificially enhancing and suppressing hippocampus-mediated memories. Curr Biol. 2019;29:1885-.e4.

72. Kiyokawa Y, Takeuchi Y. Social buffering ameliorates conditioned fear responses in the presence of an auditory conditioned stimulus. Physiol Behav. 2017;168:34-40.

73. Hall BS, Romeo RD. The influence of poststress social factors on hormonal reactivity in prepubertal male rats. Dev Psychobiol. 2014;56:1061-9.

74. Romeo RD. Perspectives on stress resilience and adolescent neurobehavioral function. Neurobiol Stress 2015;1:128-33.
75. Sterley T-L, Baimoukhametova D, Füzesi T, Zurek AA, Daviu N, Rasiah NP, et al. Social transmission and buffering of synaptic changes after stress. Nat Neurosci. 2018;21:393-403.

76. Adhikari A, Lerner TN, Finkelstein J, Pak S, Jennings JH, Davidson TJ, et al. Basomedial amygdala mediates top-down control of anxiety and fear. Nature 2015;527:179-85.

77. Sabihi S, Dong SM, Maurer SD, Post C, Leuner B. Oxytocin in the medial prefrontal cortex attenuates anxiety: anatomical and receptor specificity and mechanism of action. Neuropharmacology 2017; 125:1-12.

78. Burkett JP, Andari E, Johnson ZV, Curry DC, de Waal FB, Young LJ. Oxytocindependent consolation behavior in rodents. Science (NY, NY). 2016;351: 375-8.

79. Guzman YF, Tronson NC, Jovasevic V, Sato K, Guedea AL, Mizukami $H$, et al Fear-enhancing effects of septal oxytocin receptors. Nat Neurosci. 2013;16: 1185-7.

80. Kiyokawa Y, Honda A, Takeuchi Y, Mori Y. A familiar conspecific is more effective than an unfamiliar conspecific for social buffering of conditioned fear responses in male rats. Behav Brain Res. 2014;267:189-93.

81. Kiyokawa Y, Kikusui T, Takeuchi Y, Mori Y. Partner's stress status influences social buffering effects in rats. Behav Neurosci. 2004;118:798-804.

82. Tottenham N. Social scaffolding of human amygdala-mPFCcircuit development Soc Neurosci. 2015;10:489-99. 